\title{
USO E EXTRATIVISMO DO ANGICO NUMA COMUNIDADE INDÍGENA NA SAVANA DE RORAIMA, NORTE DA AMAZÔNIA BRASILEIRA
}

\section{USE AND EXTRACTIVISM OF ANGICO IN AN INDIGENOUS COMMUNITY IN THE RORAIMA SAVANNAH, NORTHERN BRAZILIAN AMAZON}

Rodrigo Leonardo Costa de Oliveira ${ }^{1}$

Sérgio Oliveira de Brito ${ }^{2}$

Luís Felipe Paes de Almeida ${ }^{3}$

Veridiana Vizoni Scudeller ${ }^{4}$

Reinaldo Imbrozio Barbosa ${ }^{5}$

RESUMO: O angico é uma árvore presente nos domínios fitogeográficos brasileiros da Mata Atlântica, Caatinga, Cerrado e Amazônia. Na região norte do Brasil existem registros nos estados do Amazonas, Pará, Acre e na região savânica de Roraima. Essa espécie é utilizada pelas comunidades indígenas para diversos fins, entre eles o uso medicinal da casca e principalmente na construção e combustível com o uso do caule. Diante disso esta pesquisa teve por objetivo analisar se a escolha dos angicos para corte seria determinada pelo volume do diâmetro, onde os indivíduos com os maiores diâmetros teriam preferência de uso por parte dos moradores da Comunidade Indígena Darora, etnia Makuxi, na região de savana do estado de Roraima. Foram realizadas entrevistas semiestruturadas em uma amostra composta por 60 moradores, aos quais 23 participantes (16 homens e sete mulheres), indicaram o angico como uma espécie útil na Comunidade. Análise estatística indicou que o extrativismo do angico está relacionado ao uso madeireiro da espécie.

Palavras-chave: Anadenanthera peregrina, uso madeireiro, ilha de mata, Comunidade indígena, conservação.
ABSTRACT: Angico is a tree present in the Brazilian phytogeographical domains of the Atlantic Forest, Caatinga, Cerrado and Amazonia. In the northern region of Brazil there are records in the Amazonas, Pará, Acre and the savannah region of Roraima state. This species is used by the indigenous communities for several purposes, among them the medicinal use of the bark and mainly in the construction and fuel with the use of the stem. The objective of this research was to analyze if the pattern of choice of individuals for the cut would be determined by the size of the stem diameter, where individuals with larger diameter would have preference of use by the inhabitants of the Darora Indigenous Community, Makuxi ethnic group, in the savannah region of the state of Roraima. Semistructured interviews were carried out in a sample composed of 60 residents, to which 23 participants (16 men and seven women) indicated the angico as a useful species in the Community. Statistical analysis indicated that the extraction of angico is related to the timber use of the species.

Keywords: Anadenanthera peregrina, woody use, forest island, Indigenous community, conservation.

1 Coordenação de Ciências Biológicas, Universidade Estadual de Roraima (rodrigo@uerr.edu.br).

2 Coordenação de Ciências Biológicas, Universidade Estadual de Roraima.

3 Instituto Insikiran de Formação Superior Indígena, Universidade Federal de Roraima.

4 Instituto de Ciências Biológicas, Universidade Federal do Amazonas.

5 Núcleo de Pesquisas de Roraima, Instituto Nacional de Pesquisas da Amazônia. 


\section{INTRODUÇÃO}

O nordeste de Roraima compreende a maior área de savana natural da Amazônia brasileira localmente conhecida como "lavrado", "campos do rio Branco" ou "campos de Roraima", abrangendo 43.000 km2 de área (Barbosa e Campos, 2011). A savana de Roraima faz parte do complexo de vegetação Rio Branco-Rupununi, que se estende da Venezuela para a República da Guiana (Barbosa e Fearnside, 2005), formando um grande mosaico de ecossistemas não-florestais (vegetação aberta com baixa densidade de árvores) e florestas florestais, Florestas de galeria e buritizais), com dominância de áreas de vegetação aberta (Barbosa et al., 2007).

Nesta enorme área de vegetação aberta, várias terras indígenas são encontradas, a qual as Terra Indígena Raposa/ Serra do Sol e a Terra Indígena São Marcos destacam-se por suas enormes proporções. As áreas florestais da savana apresentam importantes espécies para uso das comunidades indígenas, como importantes fontes alimentícia e medicinal, bem como de uso madeireiro para fins de construção, combustível, tecnologias e outros. Dentre estas espécies, encontra-se a Anadenanthera peregrina (L.) Speg. (Fabaceae).

A Anadenanthera peregrina faz-se presente nos domínios fitogeográficos da Mata Atlântica, Caatinga, Cerrado e Amazônia. Na região norte do Brasil, existem registros de nos estados do Amazonas, Pará, Acre e na região savânica de Roraima (Morim, 2013). No interior do estado de São Paulo, ela é caracterizada como uma espécie colonizadora e comum na vegetação secundária, podendo ser encontrada em altas densidades formando “angicais". Também pode em vegetação primária (Durigan et al., 1997; Carvalho, 2003).
Este estudo teve por objetivo analisar se a escolha dos angicos para corte seria determinada pelo volume do diâmetro, onde os indivíduos com os maiores diâmetros teriam preferência de uso em detrimento aos indivíduos de diâmetros menores.

\section{MATERIAL E MÉTODOS}

A Terra Indígena de São Marcos (TISM) compreende cerca de 654.110ha e 42 comunidades indígenas das etnias Makuxi, Taurepang e Wapixana. O presente estudo envolveu um grupo da etnia Makuxi da comunidade Darora, que vive em uma região conhecida como Baixo São Marcos ( $3^{\circ} 10^{\prime} 42$ "N; 60 23'34" W). A área de estudo fica a 90 km da capital do estado, Boa Vista. O clima local é a savana tropical (Aw), de acordo com a classificação Köppen, com uma temperatura média anual de $27,8^{\circ} \mathrm{C}$ e uma precipitação média anual de $\sim 1,650 \mathrm{~mm}$. O período mais seco ocorre entre os meses de dezembro e março $( \pm 9 \%$ de precipitação anual), e os meses mais úmidos estão entre maio e agosto ( $\pm 70 \%$ de precipitação anual) (Barbosa, 1997). A comunidade Darora tem uma forte aptidão para a agricultura e pecuária animal, entendida por eles como necessária para alimentação e sustento. O extrativismo ainda é usado e está sempre relacionado à construção de casas, cercas e coleta de frutas.

De acordo com o posto de saúde da Comunidade, 184 pessoas de 40 famílias vivem em Darora, das quais 50 são homens e 43 mulheres com mais de 18 anos; e cinco são homens e seis são mulheres com mais de 60 anos de idade. Os residentes citaram um total de nove homens e 12 mulheres, mas não foram encontrados, já que muitos deles se mudam da comunidade para a cidade de Boa Vista, buscando emprego e educação contínua. Sete residentes não foram incluídos na pesquisa, já que são Makuxi da República da Guiana e recentemente se estabeleceram na comunidade. Apenas uma mulher se 
recusou a participar. Todos os residentes falam fluentemente o português. A língua Makuxi, do tronco lingüístico Karib, é ensinada na pré-escola, mas raramente é falada pelos moradores, com apenas dois professores e um falante mais velho.

\section{Levantamento etnobotânico}

Para a coleta de dados etnobotânicos foram realizadas entrevistas semiestruturadas com 60 informantes (36 homens e 24 mulheres) entre 18 e 84 anos de idade. Foram visitadas todas as residências na comunidade, onde os moradores presentes eram convidados a participar da pesquisa. Neste momento, foi explanado sobre a finalidade da pesquisa e apresentado o Termo de Consentimento Livre e Esclarecido (TCLE) que foi assinado por todos. Na entrevista, os informantes foram direcionados a indicar o conhecimento e usos atribuído à espécie.

As entrevistas foram realizadas entre novembro de 2014 e novembro de 2015. Num primeiro momento, os entrevistados indicaram o locais de coleta das espécies estudadas em áreas não florestais de savanna, e então a técnica de lista livre foi usada para as espécies que eles conheciam na sua nesses aambientes (Albuquerque et al., 2014). O angico foi uma espécie que se destacou nas entrevistas. Em outro momento, foram realizadas entrevistas semiestruturadas com perguntas relacionadas à coleta, formas de uso, e partes das plantas utilizadas. As respostas foram agrupadas nas categorias de uso adaptadas de estudos anteriores (Lins Neto et al., 2008; Lucena et al., 2012).

\section{Disponibilidade local}

Numa ilha de mata indicada pela comunidade como local de retirada de recursos vegetais, foi instalada uma parcela de $250 \mathrm{~m} 2$, subdividida em 10 subparcelas de $25 \times 10 \mathrm{~m}$, onde foram registrados os indivíduos íntegros e cortados de Anadenanthera peregrina. Também foi realizado o método do caminhamento por todo o ambiente com o intuito de enriquecer o registro de indivíduos da espécie. Os indivíduos íntegros foram mensurados ao diâmetro à altura do peito (DAP $\geq 10 \mathrm{~cm}$ ), e os indivíduos cortados, ao diâmetro à altura de $2 \mathrm{~cm}$ do solo (DAS2 $\mathrm{cm} \geq 2 \mathrm{~cm}$ ) (Barbosa et al. 2005). A média dos indivíduos foi calculada, e foi utilizado o teste $\mathrm{T}$ para verificar diferenças entre os diâmetros dos indivíduos íntegros e cortados.

A amostra da espécie foi coletada, e após processo de herborização foi tombada no Herbário da Universidade Federal de Roraima (UFRR), sob o número de tombo 8475 (Figura 1). A identificação ocorreu com o auxílio de guias de flora da região (Melo e Barbosa, 2007; Flores e Rodrigues, 2010).

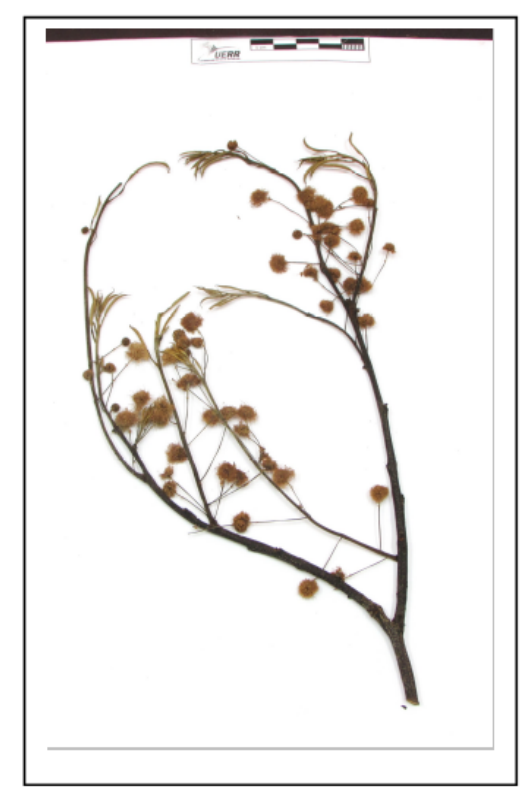

Figura 1: Angico [Anadenanthera peregrina (L.) Speg.] coletado na Comunidade Darora, Boa Vista, Roraima. Fonte: Oliveira et al. (2017).

\section{Contexto legal da pesquisa}

Este estudo é parte do projeto de pesquisa "Uso e conservação dos recursos vegetais de comunidades indígenas no norte de Roraima”, e foi submetido e aprovado pelo 
Comitê de Ética em Pesquisa com Seres Humanos do Instituto Nacional de Pesquisas da Amazônia (CEP-INPA/CONEP) sob o número 814.370; e autorizado pela Fundação Nacional do Índio (FUNAI): Processo 08620.002869/2014-15 e pelo Instituto do Patrimônio Histórico e Artístico Nacional (IPHAN): Processo 01450.001678/2014-88.

\section{RESULTADOS E DISCUSSÃO}

Apenas 23 participantes (38\%), onde 16 foram homens e sete foram mulheres, indicaram o angico como uma espécie útil na Comunidade Darora. Apesar do número de informantes ser inferior à metade do número de entrevistados (23/60), o angico se demonstrou uma espécie de bastante utilidade, uma vez que os participantes indicaram 12 usos, nas categorias tecnologia (16\% das citações de uso), combustível (2\%) e principalmente, nas categorias medicinal (24\%) e construção (58\%) (Tabela 1). O fato de poucos informantes terem indicado a espécie, pode estar associado à grande distância da ilha de mata, onde se encontram os angicos, da maioria das residências da Comunidade. No entanto, o grande número de usos associados à espécie faz dela uma das mais importantes.

Os usos associados às categorias tecnologia, combustível e construção foram indicadas principalmente pelos homens mais velhos e à categoria medicinal pelas mulheres de modo geral. Fato que entra em acordo com Voeks (2007), onde as mulheres, por apresentarem atividades cotidianas relacionadas à rotina doméstica estariam mais relacionadas às espécies medicinais e alimentício, enquanto os homens, que executam suas atividades na área externa, estariam ligadas ao uso madeireiro das espécies em ambientes áridos e semiáridos.

Os principais usos associados ao angico na categoria construção foram quanto ao uso do caule para confecção de estacas para casa ou cerca (27 citações das 50 totais) (Tabela 1). Quanto ao uso medicinal, a casca e entrecasca compuseram juntas 12 citações de uso. O que ratifica a importância medicinal da espécie que é primariamente de uso madeireiro, caracterizando uma espécie de múltiplos usos (Tabela 1). Os usos medicinal e madeireiro de Anadenanthera peregrina (L.) Speg. também foram registrados em estudos realizados em área de Cerrado e Floresta Atlântica (Pereira et al., 2012; Fernandes et al., 2014), onde a espécie era empregada na confecção de mourões, cercas, móveis, além de lenha e fabrico de remédios (Durigan et al., 1997; Carvalho, 2003). Na Caatinga, Monteiro et al (2005), também relataram usos madeireiros e medicinais para o angico (Anadenathera colubrina (Vell.) Brenan. As sementes de Anadenanthera peregrina são relacionadas ao preparo do rapé (уоро), alucinógeno usado pelos curandeiros da etnia Piaroa no sudeste da Venezuela (Báez et al., 2000).

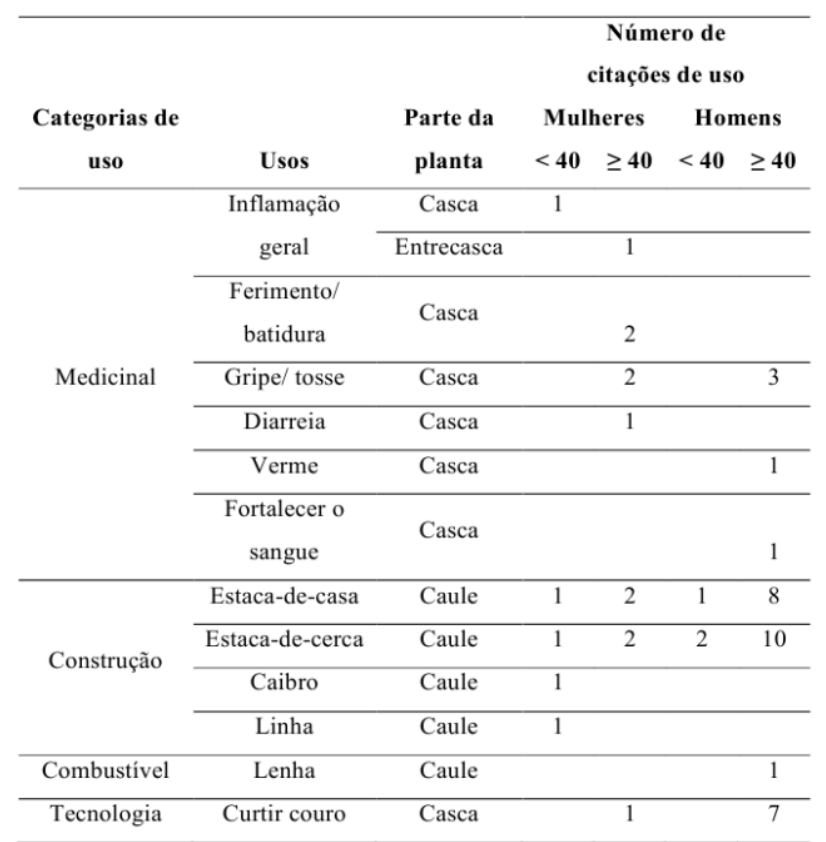

Tabela 1: Número de citações de uso de homens e mulheres quanto ao uso do angico [Anadenanthera peregrina (L.) Speg.] na Comunidade Darora, Boa Vista, Roraima. Número de participantes: 23 (16 homens e sete mulheres). 
Quanto à disponibilidade do angico na ilha de mata indicada pela comunidade, foram mensurados 13 indivíduos cortados, com média diamétrica igual à $21,8 \mathrm{~cm}$, e 18 indivíduos íntegros com média diamétrica de 13,2 cm (Figura 2). A média em altura dos indivíduos íntegros foi de 7,9 m.

$\mathrm{O}$ teste $\mathrm{T}$ indicou existir diferenças significativas, $\mathrm{p}$ (unilateral) $=0,0028$, entre os diâmetros dos indivíduos cortados em relação aos íntegros, indicando que a escolha dos indivíduos de angico são determinados pelo tamanho do diâmetro, quanto maior for o diâmetro mais suscetível ao uso madeireiro ele está pelos moradores da Comunidade Darora. A contar pelos os indivíduos mensurados nesta pesquisa, acreditamos na necessidade de realizar um estudo quanto à dinâmica populacional da espécie, com o intuito de verificar o recrutamento de novos indivíduos no local.

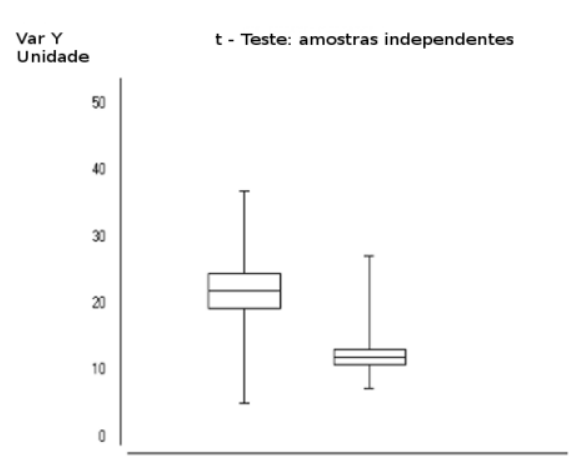

Figura 2: Distribuição diamétrica de indivíduos cortados (esquerda) e íntegros (direita) do angico [Anadenanthera peregrina (L.) Speg] na Comunidade Darora, Boa Vista, Roraima.

\section{CONCLUSÕES}

A comunidade apresenta conhecimento sobre o uso do angico [Anadenanthera peregrina (L.) Speg.] e sua coleta para fins madeireiros estão relacionados aos indivíduos com maiores dimensões diamétricas.

\section{AGRADECIMENTOS}

À Comunidade Darora e à Coordenação de Aperfeiçoamento de Pessoal de Nível Superior (CAPES), pelo empenho de uma bolsa de estudos ao primeiro autor.

\section{REFERÊNCIAS}

ALBUQUERQUE, U.P.; LUCENA, R.F.P.; LINS-NETO, E.M.F. Selection of research participants. In: Methods and techniques in Ethnobiology and Ethnoecology. Edited by Albuquerque, U.P.; Cunha, L.V.F.C.; Lucena, R.F.P.; Alves, R.R.N. Springer Protocols Handbooks, New York, p. 1-13. 2014.

BÁEZ, H., CASTRO, M.M., BENAVENTE, A.M., KINTZ, P., CIRIMELE, V., CAMARGO, C., THOMAS, C. Drugs in prehistory: chemical analysis of ancient human hair. Forensic Science International 108, 173-179. 2000.

BARBOSA, R.I. Distribuição das chuvas em Roraima. In: Barbosa, R.I., Ferreira, E.J., Castellon, E.G. (eds.) Homem, Ambiente e Ecologia no Estado de Roraima. Instituto Nacional de Pesquisas da Amazônia. Manaus, Amazonas, p. 325 - 335. 1997

BARBOSA, R.I.; CAMPOS, C. Detection and geographical distribution of clearing areas in the savannas ('lavrado') of Roraima using Google Earth web tool. Journal of Geography and Regional Planning, 4: 122-136. 2011.

BARBOSA, R.I.; CAMPOS, C.; PINTO, F.; FEARNSIDE, P.M. The "Lavrados" of Roraima: Biodiversity and Conservation of Brazil's Amazonian Savannas.

Functional Ecosystems and Communities 1(1): 29-41. 2007.

BARBOSA, R.I.; FEARNSIDE, P.M. Fire frequency and area burned in the Roraima savannas of Brazilian Amazonia. Forest ecology and management, 204: 371-384. 2005.

BARBOSA, R.I.; NASCIMENTO, S.P.; AMORIM, P.A.F.; SILVA, R.F. Notas sobre a 
composição arbóreo-arbustiva de uma fisionomia das savanas de Roraima, Amazônia Brasileira. Acta Botanica Brasilica, 19: 323-329. 2005.

CARVALHO, P. E. R. Espécies arbóreas brasileiras. Colombo: Embrapa-CNPF; Brasilia, D.F.: Embrapa-SPI, 2003. 1039p.

DURIGAN, G.; FRANCO, G. A. D. C.; PASTORE, J. A.; AGUIAR, O. T.

Regeneração natural da vegetação de cerrado sob floresta de Eucalyptus citriodora. Revista do Instituto Florestal 9(1): 7185. 1997.

FERNANDES, J. M.; GARCIA, F. C. P.; AMOROZO, M. C. M.; SIQUEIRA, L. C.; MAROTTA, C. P. B.; CARDOSO, I. M. Etnobotânica de Leguminosae entre agricultores agroecológicos na Floresta Atlântica, Araponga, Minas Gerais, Brasil. Rodriguésia 65(2): 539-554. 2014.

FLORES A.S.; RODRIGUES R.S.

Diversidade de Leguminosae em uma área de savana do estado de Roraima, Brasil. Acta Botanica Brasilica, 24: 175-183. 2010.

LINS NETO, E.M.F.; RAMOS, M.A.; OLIVEIRA, R.L.C.; ALBUQUERQUE, U.P.

The Knowledge and harvesting of Myracrondruon urundeuva Allemão by Two Rural Communities in NE Brazil. Functional Ecosystems and Communities, 2: 6671. 2008.

LUCENA, R.F.P.; MEDEIROS, P.M.; ARAÚJO, E.L.; ALVES, A.G.C.; ALBUQUERQUE, U.P. The ecological apparency hypothesis and the importance of useful plants in rural communities from Northeastern Brazil: an assessment based on use value. Journal of Enviromental Management, 96:106-115. 2012.

MELO, M.C.; BARBOSA, R.I. Árvores e arbustos das savanas de Roraima - Guia de Campo Ilustrado. $1^{\text {a }}$ ed. PMBV/CONSEMMA, Boa Vista, 36p. 2007.

MONTEIRO, J.M.; ALMEIDA, C.F.C.B.R.; ALBUQUERQUE, U.P.; LUCENA, R.F.P.;
FLORENTINO, A.T.N.; OLIVEIRA, R.L.C. Use and traditional management of Anadenanthera colubrina (Vell.) Brenan in the semi-arid region of northeastern Brazil. Journal of Ethnobiology and Ethnomedicine, 2:1-7. 2006.

MORIM, M.P. 2015 Anadenanthera in Lista de Espécies da Flora do Brasil. Jardim Botânico do Rio de Janeiro. Disponível em:

$<$ http://floradobrasil.jbrj.gov.br/jabot/floradobras il/FB22783>. Acesso em 03/09/2017.

OLIVEIRA, R. L. C.; OLIVEIRA, S. K. S.; SCUDELLER, V. V.; BARBOSA, R. I. Árvores úteis da Comunidade Darora. UERR Edições. 68p. 2017.

PEREIRA, Z. F.; FERNANDES, S. S. L.; SANGALLI, A.; MUSSURY, R. M. Usos múltiplos de espécies nativas do bioma Cerrado no Assentamento Lagoa Grande, Dourados, Mato Grosso do Sul. Revista Brasileira de Agroecologia 7(2): 126-136 2012.

VOEKS, R.A. Are women reservoirs of traditional plant knowledge? Gender, ethnobotany and globalization in northeast Brazil. Singapore Journal of Tropical Geography, 28: 7-20. 2007. 